\title{
EFEITO DO CONSERVADORISMO CONTÁBIL NA VELOCIDADE DO AJUSTE DO CAIXA
}

\author{
ACCOUNTING CONSERVATISM EFFECT ON SPEED OF ADJUSTMENT OF THE CASH
}

\author{
CRISTIANE CANTON \\ Universidade Regional de Blumenau - Mestre em Ciências Contábeis \\ Orcid: https://orcid.org/0000-0002-4074-435X / E-mail: cantoncristiane@gmail.com \\ Rua Antônio da Veiga, 140 - Itoupava Seca, CEP 89.030- 903, Blumenau - SC.

\section{MATEUS MÜLLER} \\ Universidade Regional de Blumenau - Mestre em Ciências Contábeis \\ Orcid: https://orcid.org/0000-0002-2861-7091 / E-mail: mateusmuller159@hotmail.com

\section{TARCÍSIO PEDRO DA SILVA} \\ Universidade Regional de Blumenau - Doutor em Ciências Contábeis e Administração \\ Professor e Coordenador do Programa de Pós-Graduação em Ciências Contábeis - FURB \\ Orcid: https://orcid.org/0000-0002-2370-791X / E-mail: tarcisio@furb.br

\section{MOACIR MANOEL RODRIGUES JUNIOR} \\ Universidade Regional de Blumenau - Doutor em Ciências Contábeis e Administração \\ Professor do Programa de Pós-Graduação em Ciências Contábeis - FURB \\ Orcid: https://orcid.org/0000-0003-0309-3604 / E-mail: mrodrigues@furb.br
}

\section{RESUMO}

Os aumentos nos níveis de caixa das empresas na última década atraíram considerável atenção da mídia, investidores e pesquisadores acadêmicos, visto as discussões sob várias lentes na literatura. Contudo, percebeu-se que as empresas retornam ao seu nível de caixa frente a fatores circunstanciais. Nota-se, assim, uma rapidez para ajustar-se ao nível de caixa "ideal" (velocidade de ajuste), pressupõe que esse fator pode depender do conservadorismo contábil dessas empresas. Portanto, este artigo tem por objetivo verificar o efeito do conservadorismo contábil na velocidade de ajuste do caixa. A amostra da pesquisa considera todas as companhias listadas na B3 (Brasil, Bolsa, Balcão) não financeiras e que continham todas as informações necessárias para a análise no período de 2014 a 2018. Os dados necessários, tanto para o cálculo do modelo econométrico da velocidade de ajuste do caixa quanto para o cálculo do conservadorismo contábil foram coletados a partir da base de dados Thomson Reuters ${ }^{\circledR}$. Assim, os principais resultados apontam que o conservadorismo contábil é benéfico a velocidade de ajuste, fazendo com que as empresas retornem mais rapidamente ao caixa ideal, motivado, principalmente, pela relação dos gestores nas decisões de políticas de caixa. A partir dos achados, o estudo contribui para o desenvolvimento não só acadêmico, mas com impacto no mercado, por tratar de questões ligadas a sobrevivência da empresa, auxiliando stakeholders na identificação da situação e avaliação da liquidez de caixa das companhias analisadas.

Palavras-chave: Velocidade de ajuste. Nível de caixa. Conservadorismo contábil. Bolsa de valores brasileira.

Data de submissão: 04/11/2019. Data de aceite: 30/11/2019. Data de publicação: 17/12/2019.

Artigo selecionado no fast track do COGECONT - International Conference in Management and Accounting, realizado na Universidade Comunitária da Região de Chapecó - Unochapecó, Chapecó - SC, de 17 a 19/10/2019. 


\begin{abstract}
Increases in corporate cash levels over the past decade have attracted considerable attention from the media, investors, and academic researchers, as discussions have been seen through various lenses in the literature. However, it was noticed that companies return to their cash level due to circumstantial factors. Thus, a speed to adjust to the "ideal" cash level (speed of adjustment), assumes that this factor may depend on the accounting conservatism of these companies. Therefore, this paper aims to verify the effect of accounting conservatism on the cash adjustment speed. The survey sample considers all companies listed on B3 (Brazilian stock exchange) as non-financial and that contained all the information necessary for the analysis in the period from 2014 to 2018. The data necessary for the calculation of the econometric velocity model Cash-flow adjustment and accounting conservatism calculations were collected from the Thomson Reuters ${ }^{\circledR}$ database. Thus, the main results indicate that accounting conservatism is beneficial to the speed of adjustment, making companies return more quickly to ideal cash, motivated mainly by the relationship of managers in cash policy decisions. From the findings, the study contributes to the development not only academic, but impacting the market, by addressing issues related to the survival of the company, assisting stakeholders in identifying the situation and assessing the cash liquidity of the companies analyzed.
\end{abstract}

Keywords: Speed of adjustment. Cash level. Accounting conservatism. Brazilian Stock Exchange.

\title{
1 INTRODUÇÃO
}

A temática referente as disponibilidades de caixa em finanças corporativas tornam-se de extrema relevância em estudos recentes, e a relação com a velocidade do ajuste do caixa tem sido uma das questões centrais na literatura (LIN et al., 2018; ORLOVA; RAO, 2018). De fato, a forma como as empresas evidenciam à meta para o caixa "ideal" é fundamental para inúmeros interessados, dentre esses, gestores e acionistas, visto que a decorrência da combinação errada das disponibilidades de caixa pode afetar severamente o desempenho e a sobrevivência da organização (MURITALA, 2012).

Frente a esse cenário, ao longo do tempo, teorias foram desenvolvidas e buscam compreender a forma como as organizações estruturam suas fontes de gestão para trabalhar as disponibilidades de caixa e, principalmente, a velocidade de ajustar-se ao caixa. Tem-se que, as empresas com déficits de caixa, classificadas e empresas com os superávits têm uma velocidade menor de ajuste, enquanto as empresas com excesso de caixa, firmas não classificadas e firmas que têm déficit financeiro se ajustam mais rapidamente à meta. No geral, as empresas têm um nível alvo de liquidez, no entanto, os custos de ajuste, bem como os custos de não-ajuste, afetam a velocidade com que as empresas se ajustam em relação à meta (ORLOVA; RAO, 2018).

Outro fator relevante, quanto a velocidade do ajuste do caixa relaciona-se a estrutura de capital uma vez que, vários estudos que tratam da estrutura de capital argumentam que as empresas se ajustam à alavancagem apenas quando o custo do não-ajuste excede os custos de ajuste (FAULKENDER et al., 2012). Um argumento semelhante poderia ser válido para a meta de liquidez de caixa. Nesse caso, uma fonte significativa de custo de ajuste pode ser os custos de transação associados à necessidade de levantar capital ou distribuir dividendos aos acionistas, a fim de trazer as disponibilidades de caixa para o nível da meta. 
Esses fatores destacam como as atividades contábeis da empresa podem ser influenciadas pela gestão que envolve as questões acerca da velocidade do ajuste de caixa. Nesse sentido, Louis, Sun e Urcan (2012) propõem o conservadorismo contábil como um fator separado e potencialmente influente, resultando em aumento do valor em dinheiro. De acordo com princípios contábeis conservadores, as empresas tendem a reconhecer suas perdas de maneira tempestiva, ao mesmo tempo em que adiam os ganhos até que as receitas sejam verificadas.

Essa prática, que é conhecida como temporização assimétrica, tem um efeito conservador nos ganhos. $O$ conservadorismo contábil na forma de perda pontual/reconhecimento de ganho atrasado é um método de mitigação de custos de agência (WATTS, 2003). A prática tem o efeito potencial de impedir que os gestores ocultem as perdas e evitem a responsabilidade manipulando os dados financeiros. Como resultado, as práticas contábeis conservadoras são vistas como ajudando a resolver custos de agência e aumentando o valor da empresa, diminuindo o potencial de menor valor em disponibilidade de caixa vinculado a decisões de excesso de investimento (LOUIS; SUN; URCAN, 2012).

No campo teórico, o efeito da velocidade do ajuste do caixa pode ser influenciado pelo conservadorismo contábil, uma vez que, para Orlova e Rao (2018) os custos de não se ajustar a meta do caixa incluem o aumento da probabilidade de dificuldades financeiras, se a empresa estiver deficitária em dinheiro, ou os retornos perdidos e os custos de agência em potencial (uso indevido da administração de caixa ou investimento sub-ótimo) se a empresa detiver excesso de caixa. Portanto, diante de todo o cenário descrito, emerge-se a seguinte questão de pesquisa: qual o efeito do conservadorismo contábil na velocidade do ajuste do caixa? Para responder à questão delineada, esta pesquisa apresenta como objetivo verificar o efeito do conservadorismo contábil na velocidade de ajuste do caixa.

Este estudo justifica-se por verificar um provável efeito na relação entre conservadorismo contábil e velocidade de ajuste de caixa, importantes discussões literárias. De acordo com Lin, et al. (2018) as empresas que adotam o conservadorismo contábil têm menor probabilidade de participar de atividades de superinvestimento, porque o reconhecimento oportuno de perdas limita a quantidade de caixa disponível para os administradores. Com isso, as empresas que detém excesso de caixa se adotam o conservadorismo contábil podem minimizar fatores negativos, pela perspectiva do investidor ao destino da disponibilidade alta de caixa, envolvida nessas questões.

Além disso, a pesquisa justifica-se ainda pelo cenário do estudo. De acordo com Benachenhou (2013), os denominados países emergentes, dentre eles o Brasil, caracterizam uma nova geografia econômica e social, que não permite mais negligenciá-los. Todavia, apesar deste evidente cenário, pesquisas sobre o tema de velocidade do ajuste do caixa se concentraram, por muito tempo, em países desenvolvidos. Portanto, ao buscar evidências sobre a temática obtém-se o avanço do conhecimento neste contexto. Ademais, Lopes (2002) evidenciou, no Brasil, que o conservadorismo contábil é uma das práticas mais relevantes das estruturas conceituais que direcionam as decisões corporativas.

Os resultados deste estudo apresentam evidências empíricas no que se refere ao efeito do conservadorismo contábil das empresas na rapidez com que se ajustam ao nível ideal de caixa. Assim, os achados apontam contribuições deste estudo no âmbito da literatura, por verificar a relação entre o conservadorismo contábil e a velocidade de ajuste do caixa. Além disso, os achados contribuem para o desenvolvimento econômico, uma vez que, os gestores bem como os stakeholders das companhias abertas podem tomar decisões a partir da análise desta pesquisa. 
EDIÇÃO ESPECIAL: COMEMORATIVA AOS 45 ANOS DOS CURSOS DE GRADUAÇÃO EM CIÊNCIAS CONTÁBEIS E ADMINISTRAÇÃO DA UNOCHAPECÓ

\section{REVISÃO DA LITERATURA}

Nesta seção são demonstradas as discussões acerca da temática da velocidade de ajuste do caixa bem como a relação com o conservadorismo contábil. Dessa forma torna-se possível apresentar a hipótese proposta da pesquisa.

\subsection{CONSERVADORISMO CONTÁBIL NA VELOCIDADE DO AJUSTE DE CAIXA}

Um grande corpo de literatura financeira investigou os determinantes de liquidez. Por exemplo, Opler et al. (1999) e Bates, Kahle e Stulz (009) constataram que o nível de caixa está positivamente relacionado ao fluxo de caixa, volatilidade do fluxo de caixa, gastos de capital, volatilidade da produção industrial e despesas de P\&D, e negativamente relacionado ao tamanho da empresa, capital de giro líquido, alavancagem, aquisição e pagamento de dividendos.

Para Opler et al. (1999) e Bates, Kahle e Stulz (2009) há vários motivos (interesse próprio dos agentes, economia de custos de transação e precaução contra problemas de financiamento) para explicar o nível de liquidez pela teoria do trade-off, que argumenta que, para maximizar a riqueza dos acionistas, os gestores devem equilibrar custos e benefícios de manter o dinheiro. Embora as reservas de caixa não gerem a taxa de retorno exigida, manter caixa elimina o custo da transação de vender outros ativos e fornece fundos para financiar investimentos se o financiamento externo for dispendioso ou indisponível. Contudo, Opler et al. (1999) argumentam que os investidores e gestores podem ver o equilíbrio adequado entre os custos e os benefícios do dinheiro de forma diferente.

Sob a lente da teoria do trade-off, se uma empresa se desvia do nível ótimo de caixa, deve retornar a esse nível relativamente rápido. A velocidade com que uma empresa retorna ao nível ideal de caixa é tradicionalmente referida como velocidade de ajuste de caixa $(\mathrm{CH}-$ SOA, sigla em inglês) e é medida pelo modelo de ajuste parcial. Vários estudos recentes apresentam resultados sobre o CH-SOA para empresas americanas (BATES; KAHLE; STULZ, 2009; GAO; HARFORD; LI, 2013; ORLOVA; RAO, 2018; VENKITESHWARAN, 2011).

Esses estudos destacam o fato de que o $\mathrm{CH}$-SOA varia de acordo com as características da empresa e mudam ao longo do tempo. Adicionalmente, na literatura de estrutura de capital, Oztekin e Flannery (2012) e Oztekin (2015) mostram que os determinantes institucionais contribuem para as diferenças na alavancagem da velocidade de ajuste do caixa. Pois, "melhores instituições reduzem os custos de transação associados ao ajuste da alavancagem de uma empresa" (ÖZTEKIN; FLANNERY, 2012, p. 88).

Nesse sentido, percebe-se que as investigações sobre a velocidade de ajuste do caixa (CH-SOA) ainda são uma área de pesquisa em desenvolvimento. Do ponto de vista dos investidores, existe um nível ótimo de maximização do valor do caixa que a empresa deve manter, e o desvio desse nível ótimo diminui o valor da empresa (FAULKENDER; WANG, 2006). Quando práticas contábeis conservadoras são empregadas, acionistas e conselho de administração é mais provável que ambos detectem projetos de investimento inadequados (por exemplo, projetos de sobreinvestimento ou valor líquido negativo presente (VPL)) e façam lobby para melhores decisões de gestão (LOUIS; SUN; URCAN, 2012; WATTS, 2003).

Em relação ao conservadorismo contábil, Louis et al. (2012) cita-se como um fator separado e potencialmente influente, resultando em aumento do valor em dinheiro. De acordo com princípios contábeis conservadores, as empresas tendem a reconhecer suas perdas de maneira tempestiva, ao mesmo tempo em que adiam os ganhos até que as receitas sejam verificadas. Em Watts (2003) conservadorismo contábil pode ser definido como a não 
antecipação de qualquer lucro, no entanto, o reconhecimento das todas as perdas devam ser antecipadas.

O Comitê de Pronunciamentos Contábeis (CPC), no dia 2 de dezembro de 2011, divulgou o termo de aprovação do Pronunciamento Conceitual Básico (R1), após, em confirmação pelo Conselho Federal de Contabilidade (CFC) na Norma Brasileira de Contabilidade (NBC TG), com mudanças que vem à proposito à estrutura conceitual para elaboração e publicação dos relatórios contábil financeiros. Uma dessas mudanças tratou de retirar a característica de prudência, ou conservadorismo, da condição de aspecto da representação fidedigna por ser inconsistente com a neutralidade, pois promove subavaliação de ativos e sobre avaliação de passivos (Pronunciamento Conceitual Básico CPC 00(R1), 2011) (KRONBAUER et al., 2017).

Conforme estudo seminal de Hendriksen (1982) o conservadorismo nos relatórios contábeis associa-se ao oportunismo dos gestores e dos consequentes riscos interligados. Assim, para Kan (1990) isso pode ser decorrente de inconsistências, arbitrariedades, disfarces e contradições, na evidenciação contábil (KRONBAUER et al., 2017).

Estudos sugerem que o conservadorismo possibilita melhora na eficiência em investimento. Dentre as melhoras, percebem-se duas visões principais, a ex ante referente a perdas em projeto de baixo desempenho. Quando há conservadorismo contábil, não existem incentivos para os gestores assumirem projetos negativo (BALL, 2001), devido as consequências ruins que isso causaria no resultado. Em contrapartida, quando a empresa não possui conservadorismo contábil, os gestores possuem incentivo para adiar resultados de projetos negativos com o intuito de reverte-lo com resultados futuros ou para serem reconhecidos pelo próximo gerente (AHMED; DUELLMAN, 2011).

A segunda visão trata-se de incentivos ex post para esconder resultados de projetos ruins. Isso ocorre com a finalidade de evitar a própria demissão (WATTS, 2003). Assim, a literatura descreve que o conservadorismo contábil auxilia no controle, na perspectiva de diretores e acionistas e para disciplinar os gestores com o intuito de que abandonem projetos negativos, a fim de evitar projetos de riscos elevados, optando por investimentos mais prudentes (AHMED; DUELLMAN, 2011). Portanto, o conservadorismo contábil atua como formas de melhorar o investimento em projetos que tragam melhores benefícios, entre eles, as decisões em torno da gestão do caixa, bem como a rapidez de ajustar-se ao nível de caixa ideal.

Na prática, a visão anterior é destacada por meio dos resultados de Bushman, Piotroski e Smith (2011) que evidenciaram que o conservadorismo contábil atua nas companhias por meio da melhora da rentabilidade de projetos e desinvestimentos mais oportunos. Além disso, revelaram que o conservadorismo é associado a potenciais reduções de superinvestimentos. Tal fato ocorre devido a haver queda em gastos de investimentos de baixo desempenho, em virtude de as perdas serem reconhecidas de forma mais assimétrica. Além disso, os autores Banker et al. (2016), afirmam que o conservadorismo contábil implica positivamente no fluxo de caixa futuro.

Dessa forma, a hipótese deste estudo é construída a partir da discussão dos benefícios do conservadorismo contábil para o caixa, por meio da melhor tomada de decisão em investimentos. Portanto, a hipótese 1 é a seguinte:

$\mathrm{H}_{1}$ : O conservadorismo contábil tem influência positiva na velocidade de ajuste do caixa. 


\section{PROCEDIMENTOS METODOLÓGICOS}

Tendo em vista o objetivo do estudo de verificar o efeito do conservadorismo contábil na velocidade de ajuste do caixa, a pesquisa busca nas companhias listadas na B3 resultados interessantes para a área. As pesquisas da área de contabilidade possuem suas particularidades e, devido a isso, são categorizadas de três maneiras: (i) quanto aos seus objetivos; (ii) quantos aos seus procedimentos e; (iii) quanto à abordagem do problema. Dessa forma, esse estudo é descritivo quanto aos seus objetivos, de procedimento documental e abordagem quantitativa.

A população da pesquisa concentra-se nas companhias abertas brasileiras, com ações negociáveis na B3 (Brasil, Bolsa, Balcão), em que atualmente possui aproximadamente 400 empresas. Após exclusão das instituições financeiras, que contam com bancos, holdings e afins, a amostra ainda passou por exclusão de empresas com falta de dados necessários para análise na pesquisa. Por último, foram eliminadas as companhias que não possuíam dados em, ao menos, três períodos. Assim, totalizou-se uma amostra com 331 observações de empresa-ano.

Os dados necessários, tanto para o cálculo do modelo econométrico do conservadorismo contábil quanto para o cálculo da velocidade de ajuste do caixa (descritos a seguir), foram coletados a partir da base de dados Thomson Reuters ${ }^{\circledR}$, sendo o período da pesquisa de 2014 a 2018.

\subsection{ESTIMANDO O CAIXA IDEAL}

A fim de analisar o efeito do conservadorismo contábil na velocidade do ajuste do Caixa, tem-se que o nível de Caixa Ideal representa o valor de Caixa esperado, notado pelos investidores como o valor de caixa suficiente para custear as obrigações operacionais do período (OPLER, et al., 1999). Tal valor é variável para cada companhia conforme a influência das características determinantes do Caixa Ideal e escolhas de gestão da organização. Para fins de estudo, o Caixa Ideal $(\mathrm{Cl})$ é representado pelo valor de Caixa atual. Assim, para efeitos de comparação, o desvio do caixa é representado na equação 01.

$$
\text { Velocidade de Ajuste }=\frac{\partial C}{\partial C I}
$$

O C representa o valor do Caixa no período anterior. O Cl representa o valor do Caixa no período atual, tido como o Ideal. Tal equação permite analisar a proporção na qual o ajuste é realizado no Caixa do período anterior ao atual. Quando o resultado deste cálculo for igual a um, a companhia foi capaz de ajustar toda a diferença existente entre o Caixa real e Ideal. A maioria dos estudos trata o SOA pela representação $\lambda$, na qual este simbolizaria o esforço realizado pelas empresas para a convergência ao Caixa Ideal.

A proporção $(\lambda)$ do desvio do Caixa está expresso na Equação 2.

$$
C_{i t}-C_{i t-1}=\beta\left(C I_{i t}-C I_{i t-1}\right)+\varepsilon_{i t}
$$

O $C_{i t}$ representa o Caixa. O $C_{i t-1}$ representa o Caixa do período anterior. O $C I_{i t}$ representa o Caixa Ideal. $O C I_{i t-1}$ representa o Caixa Ideal do período anterior. $O \beta$ da equação 02 representa a proporção do Caixa que se desviou do alvo do período anterior para o atual. 
O $\beta=1$ indica que a empresa realizou o desvio completo em direção ao alvo, o que, devido a custos de ajustes, espera-se que seja abaixo de 1.

Além disso, o método escolhido é o Método Generalizado de Momentos (GMM), é escolhido porque utiliza de técnicas mais complexas para isolar informações úteis e faz menos suposições sobre o processo gerador de dados subjacentes, criando um processo dinâmico com realizações atuais da variável dependente influenciada por variáveis passada (ROODMAN, 2009). Tal afirmação pode ser notada nos estudos de Venkiteshwaran (2011) e Orlova e Sun (2018), em que esse método também foi utilizado.

Com o objetivo de fortalecer o modelo, seguiu-se indicações de Roodman (2009), criando Dummies de Tempo, com o intuito de resguardar o modelo contra distúrbios idiossincráticos de indivíduos em que não assumem correlação nos testes, e Desvios Ortogonais, de forma a maximizar o tamanho da amostra nos casos em que há lacunas temporais nos painéis, tal como este estudo.

Referente as características determinantes de caixa, Bates, Kahle e Stulz (2009) e Orlova e Sun (2018) elencam variáveis que podem ser utilizadas. Dentre eles, para este estudo, foram selecionadas Market-to-Book (MTB), Tamanho, CAPEX e Capital de Giro Líquido (NWC). $\mathrm{O} M B$ é o valor de mercado dos ativos, definido como o total de ativos menos o valor patrimonial contábil mais o valor de mercado do patrimônio líquido, dividido pelo total de ativos. A relação esperada por meio dessa variável é positiva (BATES; KAHLE; STULZ, 2009).

Quadro 1 - Variáveis do Estudo

\begin{tabular}{|c|c|c|c|c|}
\hline Variável & Indicador & Fórmula & Descrição & Autores \\
\hline Caixa & C & $\frac{C_{i j}+E_{q C} C_{i j}}{A L_{i j}}$ & $\begin{array}{c}\text { Caixa }(\mathrm{C}) \text { e equivalentes de caixa } \\
\text { investimentos de curto prazo }(\mathrm{EqC}) \text {, } \\
\text { dividido pelos ativos líquidos }(\mathrm{AL}) \text {, no ano } \\
i \text { da empresa } j \text {. }\end{array}$ & \multirow{6}{*}{$\begin{array}{l}\text { Huang e } \\
\text { Mazouz } \\
\text { (2018) }\end{array}$} \\
\hline \multicolumn{4}{|c|}{ Características Determinantes do Caixa } & \\
\hline $\begin{array}{l}\text { Market-To- } \\
\text { Book }\end{array}$ & MTB & $\frac{(\mathrm{VA} \times \mathrm{QA})_{\mathrm{ij}}}{\mathrm{PL}_{\mathrm{ij}}}$ & $\begin{array}{l}\text { Valor da cotação da ação (VA) pela } \\
\text { quantidade de ações (QA)sobre o } \\
\text { Patrimônio Líquido consolidado (PL), no } \\
\text { ano } i \text { da empresa } j .\end{array}$ & \\
\hline CAPEX & CAPEX & $\frac{\mathrm{CAPEX}_{\mathrm{ij}}}{\mathrm{AT}_{\mathrm{ij}}}$ & $\begin{array}{c}\text { Relação entre gastos com ativo } \\
\text { imobilizado e AT, no ano i da empresa j; }\end{array}$ & \\
\hline $\begin{array}{l}\text { Capital de } \\
\text { Giro } \\
\text { Líquido }\end{array}$ & CGL & $\frac{\mathrm{CGL}_{\mathrm{ij}}}{\mathrm{AL}_{\mathrm{ij}}}$ & $\begin{array}{l}\text { Capital de giro líquido (CGL) dividido } \\
\text { pelos } A L \text {, no ano } i \text { da empresa } j \text {. }\end{array}$ & \\
\hline $\begin{array}{l}\text { Logaritmo } \\
\text { Natural do } \\
\text { Ativo Total }\end{array}$ & TAM & $\operatorname{Ln}(\mathrm{AT})_{i j}$ & $\begin{array}{l}\text { Tamanho representado por Ln dos ativos } \\
\text { totais (AT), no ano } i \text { da empresa } j \text {. }\end{array}$ & \\
\hline
\end{tabular}

Obs1.: Ativo líquido = total dos ativos - caixa e equivalentes de caixa; e, Obs2.: as variáveis designadas como "propostas pelos autores", referem-se, exclusivamente, para a configuração metodológica adotada nesta pesquisa. Seu uso em outras configurações é de conhecimento da literatura prévia, sobretudo na área de finanças.

Fonte: Dados da pesquisa.

Quanto ao Tamanho, essa variável tem como sua premissa a relação positiva, ou seja, quanto maior a companhia, maior é o Caixa esperado. Essa variável é calculada por meio do logaritmo natural do valor contábil do total de ativos. O CAPEX, despesa de capital, possui duas vertentes na literatura, em que são discutidos seus efeitos positivos e negativos no Caixa. O primeiro trata de possíveis custos financeiros de emergência e/ou oportunidades de 
investimento, fazendo com que a empresa opte por possuir dinheiro em caixa nos casos de tais eventos. O segundo retrata as despesas de capital como criadores, por meio dos ativos, de instrumentos a serem utilizados como garantias, diminuindo a necessidade de Caixa (BATES; KAHLE; STULZ, 2009). Por fim, o NWC é visto por meio de uma relação negativa, esperando-se que essa variável diminua a necessidade de Caixa.

A partir do Quadro 1, e com as variáveis definidas, tendo em vista a relação do Conservadorismo Contábil parte essencial desta pesquisa, torna-se necessário o cálculo do Conservadorismo Contábil, conforme demonstra-se no tópico subsequente.

\subsection{CONSERVADORISMO CONTÁBIL}

Conforme a literatura, o Conservadorismo Contábil possui uma tendência de influenciar o valor do contábil do patrimônio líquido e do lucro líquido. Apesar disso, frequentemente as citações dessa variável são restritivas e concentradas em outras formas de conservadorismo, tais como podem ser encontrados na literatura referência a contabilidade de depreciação acelerada, dispêndio de gastos com pesquisa e desenvolvimento ou avaliação de estoque (AHMED et al., 2002). Porém, a utilização do modelo de Basu (1997), posteriormente aprimorado por Nguyen et al. (2018), busca-se o conservadorismo mais amplo e que reflete seus efeitos nas escolhas contábeis, não somente escolhas de método contábil. A seguir, o modelo de Nguyen et al. (2018) é expresso na equação 3.

$$
\begin{gathered}
G A N_{i, t}=\beta_{0}+\beta_{1} D_{i, t}+R_{i, t}\left(\mu_{1}+\mu_{2} T A M_{i, t-1}+\mu_{3} M T B_{i, t-1}+\mu_{4} A L A V_{i, t-1}\right)+D_{i, t} * \\
R_{i, t}\left(\gamma_{1}+\gamma_{2} T A M_{i, t-1}+\gamma_{3} M T B_{i, t-1}+\gamma_{4} A L A V_{i, t-1}\right)+\left(\delta_{1} T A M_{i, t-1}+\delta_{2} M T B_{i, t-1}+\right. \\
\left.\delta_{3} A L A V_{i, t-1}+\delta_{4} D_{i, t} * T A M_{i, t-1}+\delta_{5} D_{i, t} * M T B_{i, t-1}+\delta_{6} D_{i, t} * A L A V_{i, t-1}\right)+\varepsilon_{i, t}
\end{gathered}
$$

Dentro desse cálculo, medidas de pontualidade foram inseridas, sendo o G_SCORE para notícias boas e C_SCORE para más notícias.

$$
\begin{aligned}
& G_{-} \operatorname{SCORE}_{i, t}=\beta 2=\mu_{1}+\mu_{2} \operatorname{TAM}_{i, t-1}+\mu_{3} M T B_{i, t-1}+\mu_{4} A L A V_{i, t-1} \\
& C_{-} \operatorname{SCORE} E_{i, t}=\beta 3=\gamma_{1}+\gamma_{2} \operatorname{TAM}_{i, t-1}+\gamma_{3} M T B_{i, t-1}+\gamma_{4} A L A V_{i, t-1}
\end{aligned}
$$

Tais estimativas das notícias são utilizadas pelo conservadorismo contábil, conforme Lara, Osma e Penalva (2016), para a o cálculo individual para cada empresa em cada ano, dando origem para a variável denominada CONS, por meio da equação 03. A partir desse valor, a classificação do grau de conservadorismo contábil se dá pela média dos últimos três períodos, o atual e mais os últimos dois das de variável CONS, o qual é ranqueado pelo e dividido por $\mathrm{N}+1$, obtendo-se uma variável com valor de 0 a 1 , representando o percentual de conservadorismo contábil da companhia.

Com os valores de Conservadorismo Contábil, o GMM ocorre a partir de dois modelos, o A, conforme equação 06, em que não se analisa o efeito do Conservadorismo no Caixa, e o modelo $B$, conforme equação 07 , com a adição dessa variável, conforme as equações a seguir.

$$
\begin{aligned}
& \text { Caixa }_{i, t}=\beta 1 \text { Caixa }_{i,-t}+\beta 2 \text { CAPEX }_{i,-t}+\beta 3 M T B_{i,-t}+\beta 4 T A M_{i,-t}+\beta 5 C G L_{i,-t} \\
& \text { Caixa }_{i, t}=\beta 1 \text { Caixa }_{i,-t}+\beta 2 \text { Caixa } \text { Conservadorismo }_{i, t}+\beta 3 \text { CAPEX }_{i,-t}+ \\
& \beta 4 M T B_{i,-t}+\beta 5 T A M_{i,-t}+\beta 6 C G L_{i,-t}
\end{aligned}
$$


Assim, para complementar o modelo GMM o Caixa defasado é utilizado como variável GMM, o efeito fixo de Setor como variável instrumental (IV) e utilizou-se de desvios ortogonais para reduzir efeito de falta de dados na amostra.

\section{ANÁLISE DOS RESULTADOS}

Nesta seção, tem-se a apresentação e análise dos resultados a partir da metodologia proposta. Assim, o primeiro passo para análise do efeito do Conservadorismo Contábil na Velocidade de Ajuste do Caixa é a análise descritiva das variáveis. Portanto, apresenta-se na Tabela 1.

Tabela 1 - Análise descritiva das variáveis.

\begin{tabular}{c|ccccc}
\hline Variável & Obs & Mínimo & Máximo & Média & Desvio Padrão \\
\hline Caixa & 331 & 0.0153 & 1.0132 & 0.3226 & 0.1262 \\
Caixa x Conservadorismo & 331 & 0.0055 & 0.5832 & 0.0926 & 0.0829 \\
Contábil & 331 & 15.0737 & 22.8654 & 19.5990 & 1.6834 \\
CAPEX & 331 & 0.1659 & 2.9370 & 0.6802 & 0.3773 \\
MTB & 331 & 20.1512 & 25.6632 & 22.7693 & 1.3476 \\
TAM & 331 & 0.0916 & 1.2507 & 0.5018 & 0.2659 \\
CGL &
\end{tabular}

Legenda: MTB - Market-to-Book; CGL - Capital de Giro Líquido; e Tam - Tamanho do Ativo Total.

Fonte: Dados da pesquisa.

Ilustra-se na Tabela 1, pela variável Caixa uma baixa dispersão da amostra, ao relacionar com as demais variáveis analisadas. Isso indica que, os valores divulgados para o caixa nas contas patrimoniais relataram que a maioria das empresas da amostra aproximam o Caixa Ideal mais próximo ao valor mínimo. Assim, tem-se que o caixa é ajustado nas empresas para o menor valor, visando alcançar a meta do caixa que tende a não manter excedentes de caixa.

Por conseguinte, ao analisar-se o Conservadorismo Contábil tem-se a multiplicação pelo Caixa, com o intuito de reduzir distorções de uma das variáveis, portanto, percebe-se uma média baixa. Isso propõe que nas empresas que adotam o conservadorismo nos relatórios contábeis não há a prática do acúmulo de caixa, assim, pode ser refletido na velocidade ao ajuste do caixa. Uma vez que, por meio do valor máximo pode se inferir que as empresas com o conservadorismo contábil tendem a ter o Caixa divulgado menor.

Em relação ao CAPEX, é possível notar a maior dispersão entre as variáveis verificadas. Saliente-se, com isso, que as empresas têm fortes gastos com o ativo imobilizado, com consideráveis diferencias entre elas. Além disso, ao avaliar a variável Market-to-Book revelase, em decorrência da média, que as companhias brasileiras são avaliadas de forma abaixo do seu valor, devido ao seu resultado ser menor do que 1. Assim, percebe-se, o contexto, com menor perspectiva de valorização e crescimento das empresas.

Quanto ao Tamanho das empresas verificadas, nota-se uma proximidade entre todas as analisadas, infere-se, assim, que os dados devem resultar numa análise adequada para os achados. Referente ao CGL, salienta-se uma baixa dispersão entre os valores de capital de giro líquido, assim, pela média destaca-se que os valores podem se assemelhar ao Caixa divulgado, indicando proximidade a um menor superávit no acúmulo de ativos líquidos.

Devido aos estudos anteriores não esboçarem as variáveis por meio de uma análise descritiva, não é possível fazer uma associação, o que seria interessante, principalmente, para as variáveis das características determinantes do caixa. Por conseguinte, avalia-se a relação de dependência entre as variáveis descritas anteriormente, para obter uma melhor 
compreensão da significância dos resultados apresenta-se a matriz de correlação entre as variáveis na Tabela 2.

Tabela 2 - Matriz de correlação (Spearman) para as variáveis da equação 2.

\begin{tabular}{l|r|r|r|r|r|c}
\hline Variáveis & Caixa & Conservadorismo & CAPEX & MTB & \multicolumn{1}{l|}{ TAM } & CGL \\
Caixa & 1,000 & 0,073 & $-0,132^{*}$ & $-0,064$ & $-0,189^{* *}$ & $0,680^{* *}$ \\
Conservadorismo & 0,073 & 1,000 & $-0,596^{* *}$ & $-0,055$ & $-0,706^{* *}$ & $0,261^{* *}$ \\
CAPEX & $-0,132^{*}$ & $-0,596^{* *}$ & 1,000 & $0,178^{* *}$ & $0,887^{* *}$ & $-0,382^{* *}$ \\
MTB & $-0,064$ & $-0,055$ & $0,178^{* *}$ & 1,000 & $0,177^{* *}$ & $-0,055$ \\
TAM & $-0,189^{* *}$ & $-0,706^{* *}$ & $0,887^{* *}$ & $0,177^{* *}$ & 1,000 & $-0,452^{* *}$ \\
CGL & $0,680^{* *}$ & $0,261^{* *}$ & $-0,382^{* *}$ & $-0,055$ & $-0,452^{* *}$ & 1,000 \\
\hline
\end{tabular}

Observação *. A correlação é significativa no nível 0,05 ( 2 extremidades); **. A correlação é significativa no nível 0,01 ( 2 extremidades).

Legenda: MTB - Market-to-Book; CGL - Capital de Giro Líquido; e Tam - Tamanho do Ativo Total.

Fonte: Dados da pesquisa.

Na Tabela 2, é possível verificar quais variáveis são significativas em sua relação, isso propõe que os resultados apresentados se tornam robustos, a fim de inferir-se resultados fidedignos a influência entre o conservadorismo contábil e a velocidade do ajuste do caixa. Assim, nota-se para o Caixa que o CAPEX, o Tamanho e o Capital de Giro líquido da empresa são significantes, e à medida que o Caixa da empresa aumenta os gastos com ativo imobilizado e o tamanho da organização tendem a diminuir, na demonstração das contas patrimoniais. No entanto, para o CGL, nota-se que a medida que o Caixa aumenta o CGL também tende a aumentar, isso indica um acumulo nos excedentes de caixa e equivalentes de caixa.

Do mesmo modo, em relação ao Conservadorismo Contábil, observa-se significância ao CAPEX e ao tamanho, e a relação demonstra que as empresas conservadoras tendem a diminuir o CAPEX e o tamanho, em contrapartida, ao capital de giro líquido. Uma vez que, para os gastos com ativo imobilizado, todas as variáveis mostram-se significantes, sendo caixa e conservadorismo negativamente significantes e tamanho, Market-to-book e CGL positivamente significantes. Isso indica, que a variável é de extrema importância e sofre influências nesse modelo de gestão para as práticas contábeis.

Quanto ao MTB, representando o valor de mercado das empresas, destaca-se porque à medida que o valor das empresas aumenta, os gastos com ativo imobilizado e o tamanho, também são maximizados. Para tanto, o tamanho das organizações mostra-se relevantes para todas as variáveis, sendo significantes com relação negativa para Caixa, Conservadorismo e Capital de giro líquido. Isso propõe que à medida que se aumenta o ativo total das empresas minimizam se os valores em caixa e equivalentes de caixa refletindo, também, no conservadorismo contábil. Todavia, para o CAPEX e o valor de mercado, nesse momento, tendem a aumentar consequentemente.

Por fim, o capital de giro líquido, mostrou-se nessa relação fortemente influenciado, uma vez que, todas as variáveis foram significantes, sendo Caixa e Conservadorismo relacionados positivamente, e CAPEX, MTB e TAM relacionados negativamente, ou seja, tendem a minimizar à medida que o CGL aumenta.

Portanto, visando compreender a relação entre o conservadorismo contábil e o a velocidade de ajuste do caixa, mostra-se em seguida, apresenta-se os resultados a partir da regressão proposta na pesquisa, para a Velocidade de Ajuste do Caixa para as companhias brasileiras, disposto na tabela 3 . 
Tabela 3 - Velocidade de Ajuste do Caixa 3.

\begin{tabular}{|c|c|c|c|c|}
\hline \multirow{2}{*}{ Variáveis } & \multicolumn{2}{|c|}{ Modelo A } & \multicolumn{2}{|c|}{ Modelo B } \\
\hline & Coef & P-V & Coef & $P-V$ \\
\hline Caixat-1 & 0.5853 & 0.000 & 0.5344 & 0.000 \\
\hline Caixa x Cons & - & - & 1.0001 & 0.030 \\
\hline CAPEX-1 & 0.0846 & 0.001 & 0.0792 & 0.002 \\
\hline MTBi $t-1$ & 0.0687 & 0.205 & 0.0033 & 0.985 \\
\hline$C_{G} L_{t-1}$ & -0.1109 & 0.001 & -0.1987 & 0.029 \\
\hline Tam $_{\mathrm{t}-1}$ & -0.0597 & 0.343 & -0.0827 & 0.028 \\
\hline Constante & 0.9836 & 0.011 & 0.4821 & 0.296 \\
\hline$\lambda$ & & $41.5 \%$ & & $46.6 \%$ \\
\hline Observações & & 331 & & 331 \\
\hline Prob > chi2 & & 0.000 & & 0.000 \\
\hline GMM & & Caixa-1 & & Caixa-1 \\
\hline IV & & Setor & & Setor \\
\hline AR (1) & & 0.000 & & 0.003 \\
\hline $\operatorname{AR}(2)$ & & 0.766 & & 0.572 \\
\hline Sargan & & 0.000 & & 0.000 \\
\hline
\end{tabular}

Legenda: Caixa; Caixa x Cons - Caixa multiplicado pelo Conservadorismo Contábil; CAPEX; MTB - Market-toBook; CGL - Capital de Giro Líquido; e Tam - Tamanho do Ativo Total. $\boldsymbol{\lambda}$ representa a velocidade de ajuste (1 lambida). Variáveis. Testes realizados para avaliar a validade do modelo do GMM System: Testes de ArellanoBond AR: (1) correlação serial de primeira ordem e (2) correlação serial de segunda ordem. Sargan de restrições de identificação excessiva.

Fonte: Dados da pesquisa.

Os modelos são significativos a nível de $1 \%$. Os testes de correlação de primeira ordem (AR 01) e de segunda ordem (AR 02) realizados indicam resultados esperados. Quanto ao teste de Sargan, o resultado é significativo, contrário ao esperado. No entanto, conforme Roodman (2009), o teste de Sargan, apesar de essenciais ao modelo, é propenso a fraqueza, o que dificulta a satisfação da hipótese nula.

Ao comparar o modelo $A$ e $B$, sendo o segundo com a variável contábil, o resultado da Tabela 3 evidencia que o Conservadorismo Contábil aumenta a velocidade de ajuste do caixa de $41,5 \%$ para $46,6 \%$. Esse achado, apesar de mais elevado, é semelhante ao de Orlova e Sun (2018), que encontraram a velocidade de ajuste para o caixa por volta de $36 \%$. Em média, as empresas fecham mais da metade de seu desvio da meta em um ano. Uma velocidade de ajuste alta é tradicionalmente interpretada como evidência de que as empresas têm um nível alvo de liquidez e se ajustam regularmente a ela, apoiando assim a teoria do trade-off de liquidez.

Esse resultado evidencia os benefícios que o conservadorismo contábil proporciona ao caixa. Conforme Louis et al. (2012), essa variável contábil atua como um fator potencialmente influente, resultando em aumento do caixa. Isso ocorre em virtude dos princípios contábeis conservadores, de reconhecer as perdas tempestivamente e adiar os ganhos, ou seja, de forma assimétrica. Ademais, Orlova e Rao (2018) revelam que o conservadorismo contábil permite a melhor no ajuste a meta do caixa, diminuindo custos de probabilidade de dificuldades financeiras e custos de agência.

Ainda, Bates, Kahle e Stulz (2009) elucida alguns fatores que resumem a importância do ajuste do caixa. Segundo os autores, há o interesse dos próprios agentes, economia de custos de transação e preocupação com problemas financeiros. Portanto, deve-se haver equilíbrio do caixa, levando em consideração os custos e benefícios de possui um nível ideal de caixa. Quanto a posição financeira da empresa. Segundo Biddle, Ma e Song (2013), o 
conservadorismo pode reduzir saída e aumentar a entrada de caixa, acarretando redução na probabilidade de notícias negativas e melhorando a posição financeira da companhia.

Referente as características determinantes do caixa, percebe-se um resultado similar ao de Orlova e Sun (2018), exceto para o Market-to-Book, que não apresentou significância. De acordo com o esperado para o capital de giro líquido (NWC), essa variável é tida como substituta do dinheiro, com isso, a sua relação esperada é negativa (BATES; KAHLE; STULZ, 2009). De acordo com a tabela $X$, denota-se o resultado esperado. Assim, percebe-se que, no contexto brasileiro, o NWC afeta negativamente o caixa.

O CAPEX, com suas duas vertentes de resultado possível, foi positivo neste estudo. Com base nisso, percebe-se que essa despesa de capital pode representar custos financeiros de emergência e, isso, relaciona-se positivamente com o caixa, devido a empresa necessitar manter dinheiro para pagamento de custos financeiros de emergência e/ou oportunidade de investimento (BATES; KAHLE; STULZ, 2009; ORLOVA; SUN, 2018). Tal resultado evidencia, para o contexto brasileiro, o CAPEX como uma preocupação quanto a emergência e/ou oportunidades, e não como uma forma de garantia de ativos para aumento da capacidade de endividamento.

Por fim, o Tamanho representa uma variável com resultado esperado como positivo, ou seja, quanto maior a companhia, mais caixa espera-se que ela possua. No entanto, percebe-se uma relação negativa. Esse resultado, apesar de adverso a literatura, também é encontrado por Bates, Kahle e Stulz (2009) e Orlova e Sun (2018). Quanto ao primeiro estudo, os autores relatam que a relação negativa entre o Caixa e o Tamanho da empresa reflete problemas de agência.

De maneira geral, percebe-se, para o contexto brasileiro, a velocidade de ajuste do Caixa como alta, $41,5 \%$ e, quando aplicado o conservadorismo contábil, a elevação para $46,6 \%$. Esse resultado permite inferir que as companhias conseguem ajustar cerca de $50 \%$ da diferença existente do seu caixa entre cada período, diminuindo problemas relacionados a custos que possam afetar os resultados e manter em um nível que proporcione os melhores benefícios.

Tal análise é importante devido ao fato de que imperfeições no mercado podem impedir a capacidade de uma empresa de gerenciar ativos líquidos fazendo com que eles se desviem dos níveis ideais (VENKITESHWARAN, 2011). Tendo em vista esse problema e outros relacionados a tomada de decisão em investimentos (LARA; OSMA; PENALVA, 2016), o Conservadorismo Contábil é uma forma de ameniza-los e atuar como potencializador da ligação com o Caixa.

Tendo em vista que a literatura traz uma relação positiva entre o conservadorismo e o dinheiro (LIN et al., 2018) e ela é vista de maneira prática neste estudo, ao aumentar a velocidade de ajuste do caixa, aceita-se a Hipótese 1 desse estudo.

\section{CONSIDERAÇÕES FINAIS}

Tendo em vista o objetivo da pesquisa que consistia em verificar o efeito do conservadorismo contábil na velocidade de ajuste do caixa, em uma amostra com 331 observações para o período em análise de 2014 a 2018 para as companhias listadas na B3. Pode-se confirmar a hipótese da pesquisa em que o conservadorismo contábil tem influência positiva na velocidade de ajuste do caixa.

Os resultados evidenciam a importância do conservadorismo contábil na análise da velocidade de ajuste do caixa. Como apontado pela literatura, essa variável contábil é capaz de beneficiar o caixa potencializando o seu aumento. Na prática, percebe-se um aumento da 
velocidade de ajuste do caixa ao se aplicar a variável de conservadorismo contábil no modelo GMM.

Infere-se, a partir do resultado do estudo, que o conservadorismo diminui os custos de ajuste, permitindo, mais facilmente, que as empresas retornem ao seu caixa ideal. Nota-se que as companhias conseguem ajustar cerca de $50 \%$ da diferença existente do seu caixa entre cada período. Isso indica que o conservadorismo diminui problemas relacionados a custos que possam afetar os resultados e mantem o caixa em um nível que proporcione os melhores benefícios para a companhia.

Este estudo traz contribuições sob várias perspectivas. Para a literatura, ao evidenciar resultados que relacionam duas temáticas de extrema relevância, uma vez que, as discussões acerca do tema da velocidade de ajuste do caixa são incipientes, tornou-se fundamental incluila em pesquisas na área de finanças corporativas, devido a essencialidade de observar seus efeitos. Pois, contribui para os estudos de Finanças Corporativas em um campo altamente fértil para discussões que permitem maximizar o desenvolvimento econômico, uma vez que as questões discutidas se inferem tanto para as companhias quanto ao mercado. Semelhante, para as empresas e seus stakeholders, como acionistas e gestores, têm-se as discussões acerca do conservadorismo contábil presente no cotidiano das práticas contábeis, podendo direcionar a tomada de decisões por meio dos achados deste estudo.

Além disso, no contexto brasileiro a relação do conservadorismo contábil e a velocidade do ajuste do caixa mostraram-se significantes e devem ser incluídos em futuras pesquisas. Diante disso, para recomendar a futuras pesquisas, nota-se que apesar de todo rigor metodológico incluído neste estudo, leva-se em consideração limitações que são necessárias avaliar para compor os resultados desta pesquisa.

Assim, relata-se a limitação de avaliar outras características dessas companhias pertencentes a amostra, que podem influenciar na velocidade do ajuste do caixa, como a governança corporativa adotada, por exemplo. Além de o estudo ser avaliado em um ponto curto no tempo, isso pode divergir em uma pesquisa com dados transversais.

Desse modo, para pesquisas futuras, cita-se a necessidade de continuar a investigação referente a velocidade de ajuste do caixa no país, como verificar o comportamento do mercado considerando empresas familiares e não familiares, para identificar referencias de gestão dependendo das particularidades das companhias. Outro ponto norteador para novas pesquisas, tem-se que em cenários desenvolvidos, como os EUA as pesquisas mostram evidências contrastantes acerca da literatura de liquidez de caixa, isso gera a necessidade de maiores investigações em diferentes contextos.

\section{REFERÊNCIAS}

AHMED, A. S.; BILLINGS, B. K.; MORTON, R. M.; STANFORD-HARRIS, M. The role of accounting conservatism in mitigating bondholder-shareholder conflicts over dividend policy and in reducing debt costs. The Accounting Review, v. 77, n. 4, p. 867-890, 2002.

AHMED, A. S.; DUELLMAN, S. Evidence on the role of accounting conservatism in monitoring managers' investment decisions. Accounting \& Finance, v. 51, p. 609-633, 2011. DOI: https://doi.org/10.1111/j.1467-629X.2010.00369.x.

BALL, R. Individualism, collectivism, and economic development. The Annals of the American Academy of Political and Social Science, v. 573, n. 1, p. 54-84, 2001. M 
BANKER, R. D.; BASU, S.; BYZALOV, D.; CHEN, J. Y. S. The confounding effect of cost stickiness on conservatism estimates. Journal of Accounting and Economics, v. 61, n. 1, p. 203-220, 2016. DOI: https://doi.org//10.1016/j.jacceco.2015.07.001.

BASU, S. The conservatism principle and the asymmetric timeliness of earnings. Journal of Accounting and Economics, v. 24, p. 3-37, 1997.

BATES, T. W.; KAHLE, K. M.; STULZ, R. M. Why do firm hold so much more cash than they used to? The Journal of Finance, v. 64, n. 5, p. 1985-2021, 2009.

BENACHENHOU, A. Países Emergentes.Brasília: Fundação Alexandre de Gusmão (FUNAG), 2013.

BIDDLE, G. C.; MA, M. L. Z.; SONG, F. M. The risk management role of accounting conservatism for operating cash flows. SSRN Electronic Journal, 2013.

DOI: https://doi.org/10.2139/ssrn.1695629.

BUSHMAN, R. M.; PIOTROSKI, J. D.; SMITH, A. J. Capital allocation and timely accounting recognition of economic losses. Journal of Business Finance \& Accounting, v. 38, n. 1-2, p. 1-33, 2011. DOI: https://doi.org/10.1111/j.1468-5957.2010.02231.x.

FAULKENDER, M.; FLANNERY, M. J.; HANKINS, K. W.; SMITH, J. M. Cash flows and leverage adjustments. Journal of Financial Economics, v. 103, n. 3, p. 632-646, 2012. Elsevier.

DOI: https://doi.org/10.1016/j.jfineco.2011.10.013.

FAULKENDER, M.; WANG, R. Corporate financial policy and the value of cash. The Journal of Finance, v. 61, n. 4, p. 1957-1990, 2006.

GAO, H.; HARFORD, J.; LI, K. Determinants of corporate cash policy: Insights from private firms. Journal of Financial Economics, v. 109, n. 3, p. 623-639, 2013. Elsevier.

DOI: https://doi.org/10.1016/j.jfineco.2013.04.008.

HENDRIKSEN, E. Accounting theory. 4 ed. Illinois: Irwin, 1982.

HUANG, W.; MAZOUZ, K. Excess cash, trading continuity, and liquidity risk Winifred. Journal of Corporate Finance, v. 48, p. 275-291, 2018. Elsevier B.V.

DOI: https://doi.org/10.1016/j.jcorpfin.2017.11.005.

KAN, V. Accounting theory. 2 ed. New York: John Wiley \& Sons, 1990.

KRONBAUER, C. A.; MARQUEZAN, L. H. F.; BARBOSA, M. A. G.; DIEHL, C. A. Análise dos efeitos do conservadorismo na informação contábil após a alteração de 2011 no pronunciamento conceitual básico. Revista Brasileira de Gestão de Negócios, v. 19, n. 65, p. 453-468, 2017. DOI: https://doi.org/10.7819/rbgn.v19i65.2742.

LARA, J. M. G.; OSMA, B. G.; PENALVA, F. Accounting conservatism and firm investment efficiency. Journal of Accounting and Economics, v. 61, n. 1, p. 221-238, 2016. Elsevier. DOI: https://doi.org/10.1016/j.jacceco.2015.07.003. 
LIN, C.; CHAN, M.; CHIEN, I.; LI, K. The relationship between cash value and accounting conservatism: The role of controlling shareholders. International Review of Economics and Finance, v. 55, p. 233-245, 2018. Elsevier Ltd.

DOI: https://doi.org/10.1016/j.iref.2017.07.017.

LOPES, A. B. A informação contábil e o mercado de capitais. São Paulo: Cengage, 2002.

LOUIS, H.; SUN, A. X.; URCAN, O. Value of cash holdings and accounting conservatism. Contemporary Accounting Research, v. 29, n. 4, p. 1249-1271, 2012.

DOI: https://doi.org/10.1111/j.1911-3846.2011.01149.x.

MURITALA, T. A. An Empirical analysis of capital structure on firms' performance in Nigeria. International Journal of Advances in Management and Economics, v. 1, n. 5, p. 116-124, 2012.

NGUYEN, T. T.; DUONG, C.; NGUYEN, N.; BUI, H. Accounting conservatism and banking expertise of boards of directors. SSRN Electronic Journal, p. 1-52, 2018. DOI:

http://doi.org/10.2139/ssrn.3101495.

OPLER, T.; PINKOWITZ, L.; STULZ, R.; WILLIAMSON, R. The determinants and implications of corporate cash holdings. Journal of Financial Economics, v. 52, p. 3-46, 1999.

ORLOVA, S. V.; RAO, R. P. Cash holdings speed of adjustment. International Review of Economics and Finance, v. 54, p. 1-14, 2018. Elsevier Ltd.

DOI: https://doi.org/10.1016/j.iref.2017.12.011.

ORLOVA, S. V.; SUN, L. Institutional determinants of cash holdings speed of adjustment. Global Finance Journal, v. 37, p. 123-137, 2018. Elsevier.

DOI: https://doi.org/10.1016/j.gfj.2018.05.002.

ÖZTEKIN, Ö. Capital structure decisions around the world: which factors are reliably important? Journal of Financial and Quantitative Analysis, v. 50, n. 3, p. 301-323, 2015. DOI: https://doi.org/10.1017/S0022109014000660.

ÖZTEKIN, Ö.; FLANNERY, M. J. Institutional determinants of capital structure adjustment speeds. Journal of Financial Economics, v. 103, n. 1, p. 88-112, 2012. DOI: https://doi.org 10.1016/j.jfineco.2011.08.01.

ROODMAN, D. How to do xtabond2: An introduction to difference and system GMM in Stata. The Stata Journal, n. 1, p. 86-136, 2009.

VENKITESHWARAN, V. Partial adjustment toward optimal cash holding levels. Review of Financial Economics, v. 20, p. 113-121, 2011. Elsevier Inc.

DOI: http://dx.doi.org/10.1016/j.rfe.2011.06.002.

WATTS, R. L. Conservatism in accounting part I: explanations and implications. Accounting Horizons, v. 17, n. 3, p. 207-221, 2003. 